19

\title{
Generalized metric based test selection and coverage measure for communication protocols
}

\author{
Jinsong Zhu and Son T. Vuong \\ Department of Computer Science \\ University of British Columbia \\ Vancouver, B.C., Canada V6T 1Z4 \\ Email: $\{j z h u, v u o n g\} @ c s . u b c . c a$
}

\begin{abstract}
This paper presents an important generalization of the metric based test selection and coverage measure, originally proposed in $[14,5]$. Although the original method introduces a significant analytical solution to the problem of coverage and test selection for protocols, its applicability is limited to only the control part of protocols. We extend this method to handle a protocol behavior space where both the control sequences and the data valuations for event parameters are included. We prove that the important properties of total boundedness and completeness are preserved in the generalized metric space, thus ensuring the possible approximation of the specification (infinite number of execution sequences) with a finite test suite within arbitrary degree of precision. A generalized test selection algorithm and coverage measure are also discussed.
\end{abstract}

Keywords

protocol testing, testing distance, test generation, coverage measure

\section{INTRODUCTION}

The handling of both the control and data parts of protocols in the process of test generation and selection has been considered as a difficult practical prob- 
lem for which no tractable analytical solution has been found. The original work on coverage measure and metric based test selection $[14,5]$ provides a significant analytical method in assessing the quality of a test suite in terms of its coverage of the specification. Contrary to fault targeting models, where detection of a predefined fault classes (also called fault model [1]) is the test purpose, this metric based method seeks to cover the behavior space of a protocol and to achieve trace equivalence of the test suite with the specification in the limit.

The metric based approach is interesting in that the metric definition with testing distance can be shown to lead to a compact metric space, and the test selection process is convergent where the more test sequences are selected the closer the selected set tends to the original set, i.e., there are no relevant, peculiar test cases or groups of test cases that may be missed out in the selection process due to mere overlook, or due to the limitation of testing cost. Furthermore, the metric defined is made general and flexible by a number of parameters which can be tuned according to the expert knowledge of the specific protocols and potential faults.

A serious limitation of the method, however, lies in the fact that it can only handle the control space of a protocol, thereby making the analysis of a large number of real life protocols infeasible. Many real life protocols have to be extended with data storage to cope with increased complexity of the protocol behavior space. The importance of data storage in practical protocols and their testing can be seen from the numerous research work on testing data flow in a protocol $[13,12,10,2,9]$. It would be technically infeasible trying to expand an extended transition system into a pure transition system by unrolling data variables into discrete values, as this would cause the well known problem of state space explosion.

In order to handle the data part feasibly and effectively, we have generalized the original metric based method (hereafter also referred to as the basic metric based or MB method). We propose a new definition of the testing distance which incorporates distance contribution from both the control elements and data values. The resulting metric space remains totally bounded and complete, which makes it possible to apply a convergent test selection process to approximate the whole protocol behavior space. A generalized test selection algorithm is proposed for this purpose, with special consideration of data variations. The coverage of the generated test set is measured by the density of the test set by using the general coverage measure as defined in the basic MB method. The extended MB method not only nicely handles protocol properties such as recursion levels, concurrent connections, and transition patterns, as supported by the basic method, but also provides a way to deal with data variations in an execution sequence. If data part is omitted, it then gracefully degrades to the basic method.

The rest of the paper is organized as follows. After a brief overview of the basic metric based method, we describe our generalized model and metric 
definition in Section 3. Section 4 proves the total boundedness and completeness properties of the generalized metric space. We then proceed to describe our test selection algorithm and coverage measure definition. We conclude by discussing the ramifications of the method and further research work.

\section{OVERVIEW OF THE BASIC METRIC BASED METHOD}

In $[14,5]$, a basic coverage metric and the basic metric based test selection method were proposed, with the purpose of generating test cases that cover the control part of the specification. The control behavior of a protocol is considered as composed of execution sequences which represent the interaction between the protocol system and its environment. Within an execution sequence, a concise notation is defined for recursive events: an event $a$ with recursion depth $\rho$ is denoted as a pair $(a, \rho)$. The control space so defined can be infinite: either an execution sequence can be infinite, or there are infinite number of execution sequences. Therefore, in order to cover the space within the computer system and time resources limit, approximations have to be made.

The basic metric based method solves this problem by defining a metric space over the behavior space made of execution sequences. A set of finite execution sequences (a test suite, or a test set) as approximations of infinite sequences, can be selected based on the metric. Furthermore, a finite number of test suites, which approximates the infinite behavior space, can be generated based on the restriction of test cost. The important property of this approximation process is that the series of test suites can converge to the original specification in the limit. Thus, we have a way to achieve coverage of the specification with an arbitrary degree of precision limited only by the test cost.

The metric is built on the concept of testing distance between two execution sequences. The distance satisfies the requirement that the resulting space be a metric space and be totally bounded, so that we have the nice property of finite covers of an infinite space [5]. It should also capture the intuitive requirement of testing relationships between execution sequences, so that a concept of "closeness" of sequences can be understood. This closeness actually represents a notion of testing representativeness: the closer the two sequences, the more likely they'll yield the same test result.

Formally, testing distance is defined as [5]:

Definition 1 (Basic testing distance) Let $s, t$ be two (finite or infinite) execution sequences in $S$, where $s=\left\{\left(a_{k}, \alpha_{k}\right)\right\}_{k=1}^{K}$, and $t=\left\{\left(b_{k}, \beta_{k}\right)\right\}_{k=1}^{L}$, $K, L \in \mathbf{N} \cup\{\infty\}$. The testing distance between two execution sequences $s$ and 
$t$ is defined as

where

$$
d t(s, t)=\sum_{k=1}^{\max \{K, L\}} p_{k} \delta_{k}(s, t)
$$

$$
\delta_{k}(s, t)= \begin{cases}\left|r_{\alpha_{k}}-r_{\beta_{k}}\right| & \text { if } a_{k}=b_{k} \\ 1 & \text { if } a_{k} \neq b_{k}\end{cases}
$$

If $s$ and $t$ are of different lengths then the shorter sequence is padded to match the length of the longer sequence, so that $\delta_{k}=1$ for all $k$ in the padded tail.

In the above definition, $\delta_{k}$ measures the difference in recursion depths. The functions $p$ and $r$ satisfy the following properties:

P1 $\left\{p_{k}\right\}_{k=1}^{\infty}$ is a sequence of positive numbers such that $\sum_{k=1}^{\infty} p_{k}=p<\infty$. This sequence defines the weights given to the differences of two events within an execution sequence.

P2 $\left\{r_{k}\right\}_{k=0}^{\infty}$ is an increasing sequence in $[0,1]$ such that $\lim _{k \rightarrow \infty} r_{k}=1$. Put $r_{\infty}=1$. This function is a normalization function that limits the distance contribution from recursion depths to $[0,1]$.

The convergence of $p_{k}$ and monotonicity of $r_{k}$ guarantee that the space $(S, d t)$ is a metric space, and more importantly it is totally bounded and complete [5, 4]. It ensures the existence of finite covers for infinite metric space $(S, d t)$, which is the theoretical foundation for the approximation process and also the test selection algorithm.

The coverage measure for a set of test sequences $T$ with respect to a set $S$ is defined as

where

$$
\operatorname{Cov}_{S}(T)=1-m_{S}(T)
$$

$$
m_{S}(T)=\frac{\sup \{d t(s, T) \mid s \in S \backslash T\}}{\sum_{k=1}^{\infty} p_{k}} .
$$

$m_{S}(T)$ represents the normalized maximum distance from $T$ to $S$. A large value of $m_{S}(T)$ implies that $T$ is "farther" from $S$, hence a smaller coverage.

\section{GENERALIZED METRIC SPACE}

The basic metric based method handles the protocol control space nicely, with particular considerations of recursion depths. However, in a general protocol behavior space where data flow plays an important role, the omission of the data part limits the applicability of the method to protocols at higher level of abstraction with data part abstracted away.

A simple-minded method to deal with data part may be to expand a protocol with data to the underlying pure finite transition system, by unrolling the 
data part into discrete values. However, this will cause the well-known state space explosion problem and is only feasible when the data part are small in number of variables and their value ranges. Moreover, even if this expansion is possible, events with different data values would be treated as totally different events, which makes the testing distance insensitive to data differences.

In order to solve this problem and broaden the applicability of the MB method, we have purported to generalize the metric space so as to accommodate both the control flow and data flow of protocols. The idea is to define one metric which incorporates distance contributions from both the execution sequences and data variations. The protocol space can thus be still considered as one metric space, and with proof of total boundedness and completeness of this space, the convergent test selection process and coverage measure can be naturally extended. Moreover, the test selection process can actually be performed in two steps: first for control sequences, and then for data variations. We show that within our distance definition, these two steps can indeed cover the whole protocol space.

We first define the concept of generalized execution sequence in our generalized protocol space, $G$, where both control flow and data flow are present. Each event now has associated data values for its parameters. A generalized execution sequence is thus a sequence of events each of which has its parameters instantiated with certain values within their respective ranges. The range for any value is assumed to be finite, which is reasonable in protocols. For recursive events, the same concise notation as in the basic method is employed. However, if the same event recurses with different data values, we do not consider as recursion depth the number of times that the event is repeated. This is because the events are essentially different when data values are different. To avoid confusion, we distinguish between an event and its name:

Definition 2 (Event) An event in an executions sequence consists of an event name, and a set of associated data values for its parameters. Formally, an event can be represented as $e\left(v_{1}, v_{2}, \ldots, v_{n}\right)$ where $e$ is the event name and $v_{1}, \ldots, v_{n}$ are a particular data valuation.

The type of the data valuations can be either numeric or alphabetic. In communication protocols, data are typically integers (e.g., sequence number) or strings (e.g., identifiers).

Definition 3 (Generalized execution sequence) A generalized execution sequence can be represented as a set of pairs: $\left\{\left(a_{k}\left(v_{1}, v_{2}, \ldots, v_{n}\right), \rho\right)\right\}$, where $a_{k}\left(v_{1}, v_{2}, \ldots, v_{n}\right)$ is an event with name $a_{k}$ and data values $v_{1}, v_{2}, \ldots, v_{n}$, and $\rho$ is the recursion depth of the event. Where ambiguity is not possible, we simply use the term "execution sequence".

All generalized execution sequences of a protocol constitutes the protocol 
space $G$. A generalized execution sequence in $G$ is also called a point in the space $G$. The testing distance between two generalized execution sequences is defined as follows:

Definition 4 (Generalized testing distance) Let $s, t$ be two (finite or infinite) generalized execution sequences in $G$, where $s=\left\{\left(a_{k}\left(u_{k 1}, \ldots, u_{k m}\right), \alpha_{k}\right)\right\}_{k=1}^{K}$, and $t=\left\{\left(b_{k}\left(v_{k 1}, \ldots, v_{k n}\right), \beta_{k}\right)\right\}_{k=1}^{L}, K, L \in \mathbf{N} \cup\{\infty\}$. The testing distance between $s$ and $t$ is defined as

$$
d t(s, t)=\sum_{k=1}^{\max \{K, L\}} p_{k} \delta_{k}(s, t)
$$

where

$$
\delta_{k}(s, t)= \begin{cases}\alpha \cdot r_{d_{k}}+\beta \cdot\left|r_{\alpha_{k}}-r_{\beta_{k}}\right| & \text { if } a_{k}=b_{k} \\ \gamma & \text { if } a_{k} \neq b_{k}\end{cases}
$$

where $\alpha, \beta, \gamma$ are constants satisfying:

$$
\alpha, \beta \geq 0, \gamma>0, \gamma \geq(\alpha+\beta) / 2,
$$

and $r_{d_{k}}$ is the vector distance between two events of the same name:

$$
r_{d_{k}}=r\left(\sum_{i=1}^{m}\left|u_{k i}-v_{k i}\right|\right)
$$

The definition of $r_{d_{k}}$ deserves a special mention in the case where the data values are alphabetic or structured. Suppose the $i$ th data value is of a general string type, i.e.,

$$
u_{k i}=p_{1} p_{2} \cdots p_{k}, v_{k i}=q_{1} q_{2} \cdots q_{k^{\prime}} \text {. }
$$

where $p_{k}, q_{k^{\prime}}$ are string elements and each has an integer value (say ASCII code for an alphanumerical element, and 0 or 1 for a bit element). Their distance can be defined as:

$$
\left|u_{k i}-v_{k i}\right|=\sum_{j=1}^{\min \left\{k, k^{\prime}\right\}}\left|p_{j}-q_{j}\right|+\left|k-k^{\prime}\right|,
$$

which is further used in the definition of $r_{d_{k}}$. The distance for structured data values, such as a record, a set, or a sequence, can be recursively computed as the sum of each component element.

In the definition of $d t(s, t)$, the functions $p$ and $r$ satisfy the same properties as in the basic metric based method (Section 2). If $s, t$ differ in length, the shorter one will be padded with a null event and $\delta_{k}=\gamma$ for the padded part.

We now prove the following theorem.

Theorem 1 The pair $(G, d t)$ is a metric space.

Proof. See appendix. 


\section{Interpretation of the distance}

We now give an intuitive interpretation of the testing distance. Same as in the basic MB method, the sequence $\left\{p_{k}\right\}_{k=1}^{\infty}$ gives the weight to the difference of two events at each position $k$. The requirement of $p_{k}$ being convergent implies that the weight should be decreasing with the increase of the length of a sequence. This is consistent with our intuition that the farther a sequence goes, the less significant the difference should be, i.e., that difference contributes less to the testing distance. Of course, one can choose to have different weights for finite number of leading events, but after that point, the weights must converge in a decreasing order. For example, a typical function $p_{k}$ is $p_{k}=1 / a^{k}(a>1)$. One may also define a $p_{k}$ as

$$
p_{k}= \begin{cases}1 & \text { if } 0<k<10 \\ 1 / a^{k} & \text { if } k \geq 10\end{cases}
$$

The function $\delta_{k}$ measures the difference in recursion depths and data variations. The purpose of using $r_{k}$ in $\delta_{k}$ is to normalize the distance contributions. $r_{k}$ is restricted in $[0,1]$ and must be increasing so that $d t$ is a distance in $G$. For example, we can choose

$$
r_{k}=\frac{a k}{a k+1} \quad \text { where } \quad a>0 .
$$

We also use the constants $\alpha, \beta$ and $\gamma$ as "fine-tuning" parameters in determining the relative importance of the difference in recursion depths, data variations, and events. As a rule of thumb, we can fine-tune the parameters as follows.

1. If difference in data variations $\left(r_{d_{k}}\right)$ is considered as more (or same, less) important than that in recursion depths $\left(\left|r_{\alpha_{k}}-r_{\beta_{k}}\right|\right)$, choose $\alpha, \beta$ such that $\alpha>\beta$ (or $\alpha=\beta, \alpha<\beta$, respectively).

2. If difference in events is considered more (or same, less) important than that in recursion depths and data variations, choose $\alpha, \beta, \gamma$ such that $\gamma>\alpha+\beta$ (or $\gamma=\alpha+\beta,(\alpha+\beta) / 2 \leq \gamma<\alpha+\beta$, respectively).

In the simplest case, we can choose $\alpha=\beta=\gamma=1$, indicating the same importance of differences in recursion depths, data variations, and actions. If we choose to neglect the data part, i.e., $\alpha=0$, and choose $\beta=\gamma=1$, we get

$$
\delta_{k}(s, t)= \begin{cases}\left|r_{\alpha_{k}}-r_{\beta_{k}}\right| & \text { if } a_{k}=b_{k} \\ 1 & \text { if } a_{k} \neq b_{k}\end{cases}
$$

which is exactly the basic metric space. 


\section{Example}

Let $a(x, y), b(z, w), c(x, w), d(y, z)$ be events, where $x, y, z$ are integers, and $w$ a character string. Consider the following three test cases:

1. $A=\{(a(x, y), 3),(d(y, z), 2),(b(z, w), 1)$,$\} .$

2. $B=\{(a(x, y), 2),(c(x, w), 2),(b(z, w), 2),(c(x, w), 1)\}$.

3. $C=\{(b(z, w), 2),(c(x, w), 3),(d(y, z), 1),(c(x, w), 2)\}$.

Using the generalized testing distance in Definition 4 with the following parameters:

$$
p_{k}= \begin{cases}2 & \text { if } 1 \leq k<2 \\ 1 / 2^{k-2} & \text { if } k \geq 2\end{cases}
$$

and

$$
r_{k}=\frac{k}{k+1}, \alpha=\beta=1, \gamma=2 .
$$

Suppose in test case $A, x=1, y=2, z=3, w=$ "DataString1", in $B, x=$ $4, y=3, z=9, w=$ "AnotherData", and in $C, x=8, y=6, z=5, w=$ "GoodTests!", we have

$$
\begin{aligned}
\delta_{1}(A, B) & =r_{d_{1}}+\left|r_{\alpha_{1}}-r_{\beta_{1}}\right| \\
& =r(3+1)+|r(3)-r(2)| \\
& =0.883 \\
\delta_{2}(A, B) & =2, \\
\delta_{3}(A, B) & =r_{d_{3}}+\left|r_{\alpha_{3}}-r_{\beta_{3}}\right| \\
& =r(6+173)+|r(1)-r(2)| \\
& =1.161, \\
\delta_{4}(A, B) & =2 .
\end{aligned}
$$

Therefore the testing distance between $A$ and $B$ is:

$$
d t(A, B)=\sum_{k=1}^{4} p_{k} \delta_{k}=7.927
$$

Similarly, we can calculate

$$
d t(A, C)=11, \quad d t(B, C)=7.119 .
$$

What this means is that, among the three test cases, $B$ and $C$ are the closest while $A$ and $C$ are the farthest. If we draw a picture with $A, B$ and $C$ as points in the protocol behavior space, they would appear as a triangle as in Figure 1. 


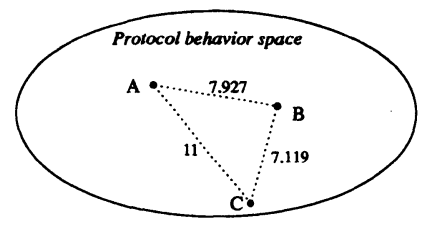

Figure 1 Testing distance between test cases A, B, and C

\section{TOTAL BOUNDEDNESS AND COMPLETENESS}

In order to be able to use finite test suite to approximate the specification (infinite number of execution sequences) with arbitrary degree of precision, we must show that the generalized metric space $(G, d t)$ is totally bounded and complete, as is the case in the basic MB method.

Theorem 2 The generalized metric space $(G, d t)$ is totally bounded.

Proof. We need to show that for every $\epsilon>0$ it is possible to cover $G$ by a finite number of spheres with radius $\epsilon$. Let $\sum_{k=1}^{\infty} p_{k}=p$ and $k_{\epsilon}$ be such that $\sum_{k=k_{\epsilon}+1}^{\infty} p_{k}<\epsilon / 2 \gamma$. Let $n_{\epsilon}$ be such that for all $k \geq n_{\epsilon}, r_{k}>1-\eta$, where $\eta=\epsilon / 2 p \beta$. $E$ is the set of all events with data valuations. Since we assume finite value range of data parameters, $E$ is finite. Therefore, the set

$$
F=\left\{\left\{\left(a_{i}\left(v_{i 1}, \ldots, v_{i n}\right), \alpha_{i}\right)\right\}_{i=1}^{k_{\epsilon}} \mid a_{i}\left(v_{i 1}, \ldots, v_{i n}\right) \in E, \alpha_{i} \in\left\{1, \ldots, n_{\epsilon}\right\}\right\}
$$

is finite.

We claim that the set of all spheres of radius $\epsilon$ centered at elements of $F$ covers the entire space. To see this, let $s=\left\{\left(b_{i}\left(u_{i 1}, \ldots, u_{i n}\right), \beta_{i}\right)\right\}_{i=1}^{L}$ be arbitrary. Then there exists $\left.t=\left\{a_{i}\left(v_{i 1}, \ldots, v_{i n}\right), \alpha_{i}\right)\right\}_{i=1}^{k_{\epsilon}}$ in $F$ such that $a_{i}=b_{i}$ and $u_{i j}=v_{i j}$ for $i=1, \ldots, k_{\epsilon}$ and $j=1, \ldots, n$. Make $\alpha_{i}=\beta_{i}$ if $\beta_{i} \leq n_{\epsilon}$ or $\alpha_{i}=n_{\epsilon}$ if $\beta_{i}>n_{\epsilon}$. Note that with this choice of $t, r_{d_{k}}(s, t)=0$ for $i=1, \ldots, k_{\epsilon}$. We have

$$
\begin{aligned}
d t(s, t) & =\sum_{k=1}^{\max \left\{k_{\epsilon}, L\right\}} p_{k} \delta_{k}(s, t) \\
& \leq \sum_{k=1}^{k_{\epsilon}} p_{k}\left(\alpha \cdot r_{d_{k}}+\beta \cdot\left|r_{\alpha_{k}}-r_{\beta_{k}}\right|\right)+\gamma \cdot \sum_{k=k_{\epsilon}+1}^{\infty} p_{k} \\
& \leq \sum_{k=1}^{k_{\epsilon}} p_{k}\left(\beta \cdot\left|r_{n_{\epsilon}}-1\right|\right)+\gamma \cdot \sum_{k=k_{\epsilon}+1}^{\infty} p_{k} \\
& \leq p \cdot \beta \eta+\gamma \cdot \epsilon / 2 \gamma \\
& <\epsilon / 2+\epsilon / 2=\epsilon .
\end{aligned}
$$


This completes our proof of the theorem.

Total boundedness is very important for the subsequent test selection because it implies the existence of finite number of balls of radius $\epsilon$ that covers the metric space. This makes it possible to choose finite number of test sequences (points in $G$ ), each belonging to one ball, to cover the protocol behavior space. The points that fall into a particular ball are all represented by the chosen point (a test sequence) in the sense that their testing distances are small enough.

The total boundedness property itself is not enough for test selection, since we want to choose test sequences incrementally, and eventually, the protocol space will be fully covered. This relies on the completeness property that we now proceed to prove. In general, we want the metric space to be compact, i.e., both totally bounded and complete.

To prove the compactness of the metric space $(G, d t)$, we need to use the following definition and preposition:

Definition 5 (Completeness) A metric space is complete if every Cauchy sequence in it is convergent to a point of the space.

Proposition 1 [11]: A metric space is compact if and only if it is complete and totally bounded.

As we have proved the boundedness of $(G, d t)$, we now only need to prove the completeness of $(G, d t)$, i.e., every Cauchy sequence in $G$ converges to a point in $G$.

Theorem 3 The generalized metric space $(G, d t)$ is complete.

Proof. Let $\left\{g_{n}\right\}_{n=1}^{\infty}$ be a Cauchy sequence in $G$, where $g_{n}=\left\{\left(a_{i}^{(n)}\left(v_{i 1}^{(n)}, \ldots, v_{i k}^{(n)}\right)\right.\right.$, $\left.\left.\alpha_{i}^{(n)}\right)\right\}_{i=1}^{K^{(n)}}, K^{(n)} \in \mathbf{N} \cup\{\infty\}$. By definition,

$$
\forall \epsilon>0 \exists N_{\epsilon}\left[\forall m, n>N_{\epsilon} \Rightarrow d t\left(g_{m}, g_{n}\right)<\epsilon\right]
$$

where $N_{\epsilon}, m, n \in \mathbf{N}$.

Let $k$ be arbitrary. We first show that $\lim _{m, n \rightarrow \infty} \delta_{k}\left(g_{m}, g_{n}\right)=0$. For an arbitrary $\eta>0$, choose $\epsilon$ such that $\epsilon<\eta p_{k}$. By definition, there exists $N_{\epsilon}$ such that $d t\left(g_{m}, g_{n}\right)<\epsilon$ for all $m, n>N_{\epsilon}$. Therefore

$$
p_{k} \delta_{k}\left(g_{m}, g_{n}\right) \leq d t\left(g_{m}, g_{n}\right)<\epsilon<\eta p_{k} .
$$

Consequently, $\delta_{k}\left(g_{m}, g_{n}\right)<\eta$ for all $m, n>N_{\epsilon}$. This proves $\lim _{m, n \rightarrow \infty} \delta_{k}\left(g_{m}, g_{n}\right)$ $=0$.

By the definition of $\delta_{k}$, we can conclude that the sequence $\left\{K^{(n)}\right\}_{n=1}^{\infty}$ must converge to $K, K \in \mathbf{N} \cup\{\infty\}$. To see this, consider two cases: a) $K^{(n)}$ are all 
finite, then $K^{(n)}$ must be eventually constant, i.e., equals $K, K \in \mathbf{N}$, since otherwise $\delta_{k}\left(g_{m}, g_{n}\right) \geq \gamma$ and cannot converge to $0 ;$ b) $K^{(n)} \rightarrow \infty$ when $n \rightarrow \infty$, in which case we also conveniently say $K^{(n)}$ converges to $\infty$.

Similarly, we can conclude that

$$
\lim _{m, n \rightarrow \infty} r_{d_{k}}=0 \quad \text { and } \quad \lim _{m, n \rightarrow \infty}\left|r_{\alpha_{k}}-r_{\beta_{k}}\right|=0
$$

This implies that the sequences $\left\{a_{i}^{(n)}\right\}_{n=1}^{\infty},\left\{v_{i k}^{(n)}\right\}_{n=1}^{\infty}$ 's, and $\left\{\alpha_{i}^{(n)}\right\}_{n=1}^{\infty}$ are all convergent. Let $a_{i}\left(v_{i 1}, \ldots, v_{i k}\right)=\lim _{n \rightarrow \infty} a_{i}^{(n)}\left(v_{i 1}^{(n)}, \ldots, v_{i k}^{(n)}\right)$ and $\alpha_{i}=\lim _{n \rightarrow \infty} \alpha_{i}^{(n)}$. Since $i$ is arbitrary, we get the sequence $g=\left\{\left(a_{i}\left(v_{i 1}, \ldots, v_{i k}\right), \alpha_{i}\right)\right\}_{i=1}^{K}, K \in$ $\mathbf{N} \cup\{\infty\}$, which is a point in space $G$.

We claim that the sequence $\left\{g_{n}\right\}_{n=1}^{\infty}$ converges to $g$. This is obvious when $K$ is finite since eventually $g_{n}=g$. To prove the case where $K=\infty$, let $\epsilon>0$ be arbitrary. Choose $k_{\epsilon}$ such that $\sum_{k_{\epsilon}+1}^{\infty} p_{k}<\epsilon / 2$. Since $\lim _{n \rightarrow \infty} \delta_{k}\left(g_{n}, g\right)=0$, we can choose $n_{\epsilon}$ such that $\delta_{k}\left(g_{n}, g\right)<\epsilon / 2 p$ (where $p=\sum_{k=1}^{\infty} p_{k}$ ) for all $1 \leq k \leq k_{\epsilon}$ and for all $n \geq n_{\epsilon}$. Therefore, for all $n>n_{\epsilon}$ we have

$$
\begin{aligned}
d t\left(g_{n}, g\right) & =\sum_{k=1}^{\infty} p_{k} \delta_{k}\left(g_{n}, g\right) \\
& \leq \sum_{k=1}^{k_{\epsilon}} p_{k} \delta_{k}\left(g_{n}, g\right)+\sum_{k=k_{\epsilon}+1}^{\infty} p_{k} \\
& <\frac{\epsilon}{2 p} \sum_{k=1}^{k_{\epsilon}} p_{k}+\frac{\epsilon}{2} \\
& \leq \frac{\epsilon}{2 p} p+\frac{\epsilon}{2}=\epsilon .
\end{aligned}
$$

This proves that the Cauchy sequence $\left\{g_{n}\right\}_{n=1}^{\infty}$ in $G$ converges to a point in $G$ with respect to $d t$, therefore $(G, d t)$ is complete.

From Theorem 2 and 3 and the Preposition, we obtain the following compactness theorem.

Theorem 4 The generalized metric space $(G, d t)$ is compact. $\square$

The compactness (i.e., total boundedness plus completeness) lays the foundation of test selection and coverage measure in the next section. We will modify the test selection process in the basic MB method to make it applicable to the generalized space. 


\section{TEST SELECTION AND COVERAGE MEASURE}

The basic idea in test selection with the basic MB method is to generate $\epsilon$-dense* ${ }^{*}$ set of test sequences with respect to the protocol specification or some original test suite. By choosing $\epsilon$ arbitrarily small and incrementally adding more test sequences, we can achieve arbitrary accuracy in covering the protocol specification in a convergent manner.

We adopt the same idea in our generalized metric space, with extensions to handle the data valuations. For simplicity, we omit the cost factor [14] in our algorithm, and focus on the selection of $\epsilon$-dense test sets with respect to a set of execution sequences which can be finite or infinite.

Algorithm: Selection of an $\epsilon$-dense set of test sequences

Input: $G$ : original set of execution sequences, $\epsilon$ : density requirement. Output: $T$ : selected $\epsilon$-dense set of test sequences.

Step 1: Initially, $T=\phi$ (empty). Also let $X=G$.

Step 2: If $X=\phi$ return $T$ and exit. Otherwise randomly remove a test sequence $t$ from $X$ (i.e., $X \leftarrow X-\{t\}$ ), apply appropriate data valuations where data are involved, and calculate $d t(t, T)$.

Step 3: If $d t(t, T) \geq \epsilon, T \leftarrow T \cup\{t\}$. Go to Step 2 .

Using the same idea of multi-pass algorithm with decreasing $\epsilon$ density, we obtain a set of Cauchy sequences over the successive passes. These Cauchy sequences converge to infinite execution sequences in the specification when $G$ is infinite. Since the space $(G, d t)$ is totally bounded, we can eventually find the finite covering of $G$ with density $\epsilon$, at which point the algorithm terminates.

It is also worthwhile to note that in Step 2 when data valuations are generated, we can either simply generate random data values, or intentionally select fault sensitive data values such as boundary values and typical values. In this way, some test data generation criteria that have been proved effective in software testing can be incorporated. In practice, we need also consider the executability of an execution sequence. This executability checking can be performed on-the-fly as data values are chosen, using the method in [3] or [9].

In the implementation of the algorithm, we may also consider all data variations for an execution sequence before proceeding to a new execution sequence, or consider all execution sequences with distinct control part before embarking on data variations. Either way we can have a more clear-cut two pass algorithm that clearly distinguishes between the control and data part,

*A set $T$ is said to be $\epsilon$-dense $(\epsilon>0)$ in a set $S$ if for every $s \in S$ there is $t \in T$ such that $d t(s, t)<\epsilon$. 
and yet they together cover the whole protocol space with a desired test set density.

From the test selection process, we can actually measure the coverage of a test set with density $\epsilon$. This can be seen by observing that

$$
\sup \{d t(g, T): g \in G \backslash T\} \leq \epsilon
$$

Using the same definition of the basic MB method (Section 2), we have

$$
m_{G}(T)=\frac{\sup \{d t(g, T): g \in G \backslash T\}}{\sum_{k=1}^{\infty} p_{k}} \leq \frac{\epsilon}{p},
$$

where $p=\sum_{k=1}^{\infty} p_{k}$. Consequently,

$$
\operatorname{Cov}_{G}(T)=1-m_{G}(T) \geq 1-\frac{\epsilon}{p} .
$$

Using $1-\epsilon / p$ as the coverage of $T$ with respect to $G$ would be a fair measure since it is the lower bound of the exact coverage. The property $\lim _{\epsilon \rightarrow 0} \operatorname{Cov}_{G}(T)$ $=1$ also indicates that with arbitrarily small $\epsilon$ density, the test set can ultimately cover the whole space.

\section{CONCLUDING REMARKS}

The problem of test generation and selection, which can handle both the control and data parts of protocols has been considered as a difficult practical problem for which no tractable analytical solution has been found. In this paper, we have presented a generalization of the basic metric based test selection method where both control and data parts of protocols can be handled. The major contributions include a generalized metric space definition, the proof of total boundedness and completeness (i.e., compactness) properties of the space, and a generalized test selection algorithm. This lays out a metric based testing framework where extended protocol space can be tackled. In our generalized test selection algorithm, it is also possible to incorporate test data generation criteria that have been proved effective in discovering program faults, such as boundary values, typical values, and values that cover data dependencies, etc. This could be a point where our metric based method is joined by the traditional software testing techniques, and therefore its effectiveness and applicability are boosted. We are currently doing experiments with practical protocols in order to show this effectiveness.

It is true that the MB method critically hinges on the definition of testing distance, and with different definitions, the effectiveness may be different. However, the testing distance is intended to provide a way to measure the "closeness" or "representativeness" of test sequences. The exact meaning of the "closeness" concept relies on our experience and expertise in protocol testing. With our definition of the testing distance, we believe many important protocol properties, such as recursion, concurrency, and data variations, are 
already captured in the definition. With appropriate parameterization of the functions $r$ and $p$, and the fine-tuning constants $\alpha, \beta$ and $\gamma$, a "good" testing distance definition which reflects our experiences in protocol testing is possible. It is of course a non-trivial task to incorporate expert knowledge into the parameters. This is actually a further research area where heuristics need to be developed to provide guidance to the choice of metric functions and parameterizations. An initial research work that evaluates the sensitivity of metric functions to recursion depths, concurrent connections, and transition patterns is reported in [6].

It is worth noting that our method is not intended as a replacement of fault targeting methods; instead we view them as complementary methods for testing protocols and software in general. In a typical test campaign, functional testing is first carried out to make sure an implementation indeed performs the functions it is supposed to perform. Structural testing follows by checking that the protocol or program space is sufficiently exercised. Our method can be very useful in the second stage in that it can systematically generate test sets with increased coverage, and the test sets converge to the whole space eventually, with the accuracy only limited by cost.

As many software testing researchers have noticed and showed with experiments that code coverage does imply fault coverage [7], we conjecture that our method should also have good fault coverage. How good it is should still depend on the definition of testing distance and possibly the structure of a protocol. We plan to explore this further with experiments, by observing and analyzing the sensitivity of metric functions to the fault detection capability of the test sets. As a final remark, we observe and conjecture that there is link between our method and software reliability which can be measured with test set density in some way. Further work will be necessary to validate (or invalidate) the conjecture.

\section{ACKNOWLEDGMENTS}

The authors wish to thank Dr. Jadranka Alilovic-Curgus for many fruitful discussions during the course of the work reported in this paper, and the anonymous reviewers for their helpful comments.

\section{APPENDIX}

Proof of Theorem 1: It suffices to show that $d t$ is a distance in $G$.

1. $d t(s, t)$ is a non-negative real number for any $s, t$.

This is straightforward from the fact that $\delta_{k}$ is up-bounded by $\max \{\alpha+$ $\beta, \gamma\}$, and the sequence $p_{k}$ converges.

2. $d t(s, t)=0$ iff $s=t$.

This can be easily seen from the fact that $d t(s, t)=0$ iff $\delta_{k}(s, t)=0$ for all 
$k$, which is equivalent to $K=L, a_{k}=b_{k}, u_{k i}=v_{k i}$ for all $i$, and $\alpha_{k}=\beta_{k}$, for all $k=1,2, \ldots, K=L$.

3. $d t(s, t)=d t(t, s)$.

This directly comes from the fact that $\delta_{k}(s, t)$ is symmetric, i.e., $\delta(s, t)=$ $\delta(t, s)$.

4. $d t(s, t)$ satisfies the triangular inequality.

Let $s, t, u$ be three execution sequences in $G, u=\left\{\left(c_{k}\left(w_{k 1}, \ldots, w_{k l}\right), \gamma_{k}\right)\right\}_{k=1}^{M}$. Without loss of generality, consider the following cases at $\delta_{k}$ :

Case I: $s, t, u$ all have the same event, i.e., $a_{k}=b_{k}=c_{k}$. We have

$$
\begin{aligned}
& \delta_{k}(s, u)+\delta_{k}(u, t) \\
= & \left(\alpha \cdot r_{d_{k}}(s, u)+\beta \cdot\left|r_{\alpha_{k}}-r_{\gamma_{k}}\right|\right)+\left(\alpha \cdot r_{d_{k}}(u, t)+\beta \cdot\left|r_{\gamma_{k}}-r_{\beta_{k}}\right|\right) \\
= & \alpha \cdot\left(r_{d_{k}}(s, u)+r_{d_{k}}(u, t)\right)+\beta \cdot\left(\left|r_{\alpha_{k}}-r_{\gamma_{k}}\right|+\left|r_{\gamma_{k}}-r_{\beta_{k}}\right|\right) \\
\geq & \alpha \cdot r_{d_{k}}(s, t)+\beta \cdot\left|r_{\alpha_{k}}-r_{\beta_{k}}\right| \\
= & \delta_{k}(s, t) .
\end{aligned}
$$

The inequality $r_{d_{k}}(s, u)+r_{d_{k}}(u, t) \geq r_{d_{k}}(s, t)$ holds due to a special case of the Minkowski's inequality [8] and also the fact that the function $r$ is increasing.

Case II: $s, t$ have the same event, but $u$ 's event is different, i.e., $a_{k}=b_{k} \neq c_{k}$. Since $\gamma \geq(\alpha+\beta) / 2$, it follows that

$$
\begin{aligned}
\delta_{k}(s, u)+\delta_{k}(u, t) & =2 \gamma \\
& \geq \alpha+\beta \\
& \geq \alpha \cdot r_{d_{k}}+\beta \cdot\left|r_{\alpha_{k}}-r_{\beta_{k}}\right| \\
& =\delta_{k}(s, t) .
\end{aligned}
$$

Case III: $s, t$ have different event, but $u$ has the same event as $s$, i.e., $a_{k}=c_{k} \neq b_{k}$. We have

$$
\delta_{k}(s, u)+\delta_{k}(u, t)=\left(\alpha \cdot r_{d_{k}}(s, u)+\beta \cdot\left|r_{\alpha_{k}}-r_{\gamma_{k}}\right|\right)+\gamma \geq \gamma=\delta_{k}(s, t) .
$$

Case IV: $s, t, u$ all have different event, i.e., $a_{k}, b_{k}, c_{k}$ are all different. Obviously,

$$
\delta_{k}(s, u)+\delta_{k}(u, t)=\gamma+\gamma>\gamma=\delta_{k}(s, t) .
$$

Therefore, in all cases, we have $\delta_{k}(s, u)+\delta_{k}(u, t) \geq \delta_{k}(s, t)$, which leads to:

$$
\begin{aligned}
d t(s, u)+d t(u, t) & =\sum_{k=1}^{\max \{K, L\}} p_{k} \delta_{k}(s, u)+\sum_{k=1}^{\max \{L, M\}} p_{k} \delta_{k}(u, t) \\
& \geq \sum_{k=1}^{\max \{K, L\}} p_{k} \delta_{k}(s, t)=d t(s, t) .
\end{aligned}
$$




\section{REFERENCES}

[1] G.v. Bochmann and et al. Fault models in testing. In J. Kroon, R.J. Heijink, and E. Brinksma, editors, Protocol Test Systems, V, Leidschendam, The Netherlands, October 1991.

[2] S.T. Chanson and J. Zhu. A unified approach to protocol test sequence generation. In Proc. IEEE INFOCOM, San Francisco, March 1993.

[3] S.T. Chanson and J. Zhu. Automatic protocol test suite derivation. In Proc. IEEE INFOCOM, Toronto, Canada, June 1994.

[4] J.A. Curgus. A metric based theory of test selection and coverage for communication protocols. PhD thesis, Dept. of Computer Science, Univ. of British Columbia, June 1993.

[5] J.A. Curgus and S.T. Vuong. A metric based theory of test selection and coverage. In Proc. IFIP 13th Symp. Protocol Specification, Testing, and Verification, May 1993.

[6] J.A. Curgus, S.T. Vuong, and J. Zhu. Sensitivity analysis of the metric based test selection. In IFIP 10th Int. Workshop on Testing of Communicating Systems, Cheju Island, Korea, September 1997.

[7] P.G. Frankl and S.N. Weiss. An experimental comparison of the effectiveness of branch testing and data flow testing. IEEE Transactions on Software Engineering, 19(8):774-787, August 1993.

[8] E. Hewitt and K. Stromberg. Real and Abstract Analysis. SpringerVerlag, 1965.

[9] T. Higashino and G.v. Bochmann. Automatic analysis and test case derivation for a restricted class of LOTOS expressions with data parameters. IEEE Transactions on Software Engineering, 20(1), January 1994.

[10] R.E. Miller and S. Paul. On generating test sequences for combined control and data flow for conformance testing of communication protocols. In Proc. IFIP 12th Int. Symp. on Protocol Specification, Testing, and Verification, June 1992.

[11] H.L. Royden. Real Analysis. Macmillan Publishing Company, New York, 1988.

[12] B. Sarikaya, G.v. Bochmann, and E. Cerny. A test methodology for protocol testing. IEEE Transactions on Software Engineering, May 1987.

[13] H. Ural. Test sequence selection based on static data flow analysis. Computer Communication, 10(5), 1987.

[14] S.T. Vuong and J.A. Curgus. On test coverage metrics for communication protocols. In Proc. 4th Int. Workshop on Protocol Testing System, 1991. 